\title{
Factors Associated with Clinical Outcomes of Differentiated Thyroid Cancer Following Radioiodine Therapy in Tanzania
}

\author{
Lulu Lunogelo Sakafu ${ }^{1,}$, , Teddy Frank Mselle ${ }^{2}$, Julius David Mwaiselage ${ }^{1}$, \\ Khamza Kibwana Maunda ${ }^{1}$, Katherine Van Loon ${ }^{3}$, Bouyoucef Salah Eddin ${ }^{4}$ \\ ${ }^{1}$ Department of Clinical Oncology, Muhimbili University of Health and Allied Sciences, Dar es Salaam, Tanzania \\ ${ }^{2}$ School of Medicine, Muhimbili University of Health and Allied Sciences, Dar es Salaam, Tanzania \\ ${ }^{3}$ Division of Hematology and Oncology, Department of Medicine, University of California, San Francisco, USA \\ ${ }^{4}$ Department of Nuclear Medicine, CHU Bab El Oued University, Algiers, Algeria
}

Email address:

willymeena2007@yahoo.com (L. L. Sakafu)

${ }^{*}$ Corresponding author

\section{To cite this article:}

Lulu Lunogelo Sakafu, Teddy Frank Mselle, Julius David Mwaiselage, Khamza Kibwana Maunda, Katherine Van Loon, Bouyoucef Salah Eddin. Factors Associated with Clinical Outcomes of Differentiated Thyroid Cancer Following Radioiodine Therapy in Tanzania. Cancer Research Journal. Vol. 7, No. 3, 2019, pp. 73-78. doi: 10.11648/j.crj.20190703.11

Received: June 3, 2019; Accepted: July 2, 2019; Published: July 23, 2019

\begin{abstract}
Background: Thyroid cancer is the most common endocrine type of malignancy, accounting for 1-5\% of all cancers worldwide. Most of the differentiated thyroid cancers are asymptomatic. Surgery is the mainstay of management to be followed by radioactive iodine (RAI). RAI accessibility is still a challenge in most developing countries including Tanzania. The aim of this study was to determine factors affecting the clinical outcome of patients with differentiated thyroid cancer (DTC) following RAI treatment in a resource limited setting. Methods: This was a prospective cohort study carried out from 2014 to 2018 at the Ocean Road Cancer Institute, in Tanzania. A total of 52 histologically proven differentiated thyroid cancer patients post- near or total thyroidectomy were recruited. All patients received RAI therapy until ablation was achieved, were maintained on thyroxine suppression dose, and were followed for two years. Results: A total of 52 differentiated thyroid cancer patients were recruited after surgery by convenience sampling. The median age of patients was 46 years (range 17-77), and $87 \%(n=45)$ were female. Distant metastases were detected in $60 \%$ of patients $(n=20)$ at initial presentation. The most common clinical presentation was a neck mass without compression symptoms (85\%). Analysis at the end of two years revealed that female gender, clinical-pathological presentation, and the absence of distant metastasis(es) at diagnosis and amount of RAI received, contributed significantly to improved outcome. Conclusion: In a limited resource setting, the outcome of DTC patients post RAI therapy can be improved by early diagnosis hence improving clinical outcome.
\end{abstract}

Keywords: Radioiodine Therapy, Thyroid Cancer, Tanzania

\section{Introduction}

Thyroid carcinoma is the most common type of malignant endocrine neoplasia, accounting for $1-5 \%$ of all cancers worldwide [1-2]. Follicular thyroid cancer (FTC) and papillary thyroid cancer (PTC) are the main subtypes and are derived from follicular epithelial cells [3]. The exact cause for their occurrence is not known, but dietary iodine deficiency has been implicated as a potential risk factor [4-5].
Most differentiated thyroid cancers (DTC) are asymptomatic and present as painless swelling in the anterior neck. Patients may also present with hoarseness, dysphagia, cough, and dyspnea, which are symptoms often associated with more advanced disease. These patients usually have cervical lymphadenopathy, or distant metastasis, commonly to the lungs or bones and rarely to the brain and liver $[3,6]$.

For patients with localized disease at presentation, the standard of care immediately after a diagnosis of a neck swelling is to perform surgery to remove the entire lesion and 
the remaining normal thyroid tissue as well as the regional lymph nodes [7]. Within six to eight weeks of surgery, patients should receive a RAI ablation dose of $100 \mathrm{mCi}(3700$ $\mathrm{mBq}$ ) [8]. By this time a patient will also be hypothyroid due to removal of entire thyroid gland hence thyroxine hormone supplementation has to be instituted soon after [9]. Thereafter, patients are followed up after every six months to reassess if the patient has attained complete ablation before repeating surgery or RAI treatment [10].

According to American Thyroid Association (ATA) [11], the clinical outcome of RAI ablation dose should be assessed 6-8 months later. Neck ultrasound is useful in detecting any residual thyroid tissue in the thyroid bed and any presence of local and regional lymphadenopathy [12]. Further diagnostic $\mathrm{I}^{131}$ whole body scan (WBS) is used to assess the completeness of ablation by detecting any residual thyroid uptake on the thyroid bed or any focus of uptake anywhere in the body which signifies recurrent or distant disease. Thyroglobulin is a tumor marker for DTC and is also used to assess any DTC focus elsewhere in the body [11-12].

DTC represent approximately $1 \%$ of cases who receive care at Tanzania's national cancer referral, Ocean Road Cancer Institute in Dar es Salaam. Currently, patients are diagnosed and undergo surgery at Muhimbili National Hospital and at other referral and regional hospitals and are subsequently referred to ORCI for RAI. We aimed to evaluate factors associated with clinical outcomes following RAI treatment in Tanzania.

\section{Methodology}

\subsection{Study Design}

This was a prospective cohort study of patients who received care for DTC at ORCI between 2014 and 2018. ORCI is the only specialized center for cancer treatment in Tanzania that offers RAI therapy and is currently home to an isolation facility for RAI treatment and two dedicated gamma cameras that are used for post RAI imaging as well as diagnostic whole body scan with $\mathrm{I}^{131}$ during follow up visits. Convenience sampling was used to recruit patients to the study cohort.

This study was approved by the Muhimbili University of Health and Allied Sciences (MUHAS) Institutional Review Board.

\subsection{Treatment Protocol}

Since ORCI does not currently offer surgical services, all surgeries for thyroid cancer are performed at Muhimbili National Hospital and other referral and regional hospitals in Tanzania. According to our institutional protocol, repeat $I^{131}$ treatments were administered at 6-8 month intervals until ablation of all functioning tumor tissue had been achieved which ware preceded by surgery whenever indicated. After total ablation, all patients were maintained on maximum tolerated thyroxine therapy.
The outcome of RAI therapy was assessed by neck ultrasound, thyroglobulin levels and $\mathrm{I}^{131}$ whole body scan at six months post ablation and then every 6-8 months. Those who had incomplete ablation after the first ablation with $\mathrm{I}^{131}$ $100 \mathrm{mCi}(3700 \mathrm{mBq})$ at six months, they underwent surgical remnant removal then received a second dose of $150 \mathrm{mCi}$ $(5550 \mathrm{mBq})$ and were re-evaluated after another six month. If a complete ablation was not attained then a third dose of either $150 \mathrm{mCi}(5550 \mathrm{mBq})$ or $200 \mathrm{mCi}(7400 \mathrm{mBq})$ depending on the site of metastasis was given post remnant surgical removal whenever accessible.

\subsection{Data Collection}

Clinicopathologic data was abstracted from the medical record, including age, gender, clinical presentation, type of surgery, and the presence or absence of distant metastasis(es) prior to RAI therapy. Treatment data, including number and reasons for repeated surgeries, dose(s) of RAI therapy, dose of thyroxine, and frequency and dose of repeated RAI therapies were abstracted from the medical records. Detailed follow-up information regarding detection of incomplete or complete ablation and the patient's vital status were collected every six months for a follow-up period of two years following initial treatment. The clinical outcomes were defined as complete remission, incomplete ablation, or death. Complete remission was defined as complete ablation six months post RAI therapy and at any six month interval during follow up. Incomplete ablation was defined as residual or disease progression.

\subsection{Statistical Analysis}

All data were entered into Excel files. Statistical Package for Social Science (SPSS) version 20 was used for all statistical analyses. Potential risk factors and clinical outcome were considered as dependent variables. Sociodemographic factors, disease profile, and treatment doses were considered as independent variables. Descriptive statistics were used. Associations between risk factors and treatment outcome are calculated by chi square.

\section{Results}

A total of 52 patients who met the inclusion criteria and consented to participate in the study were recruited.

\subsection{Sociodemographic and Clinical Characteristics of Study Participants}

As summarized in Table 1 the median age of patients was 46 years (range 17-77), 87\% of patients were female, and papillary carcinoma was the most common pathologic subtype (56\%). Patients with a follicular subtype were more likely than patients with papillary or Hurthle cell subtypes to have distant metastases at time of diagnosis (60\% vs. $30 \%$ vs. $0 \%, \mathrm{p}=0.02$ ). 
Table 1. Demographic and Clinical Characteristics of Patients at Presentation $(N=52)$.

\begin{tabular}{|c|c|c|c|c|c|}
\hline \multirow{2}{*}{ characteristics } & \multicolumn{3}{|c|}{ Histological subtypes } & \multirow{2}{*}{ Total } & \multirow{2}{*}{$p$-value } \\
\hline & Follicular & Papillary & Hurthle cell & & \\
\hline \multicolumn{6}{|l|}{ Age } \\
\hline$>45$ & 13 & 12 & 1 & 26 & \\
\hline$<45$ & 7 & 17 & 2 & 26 & 0.2 \\
\hline \multicolumn{6}{|l|}{ Sex } \\
\hline Male & 4 & 3 & 0 & 7 & \\
\hline Female & 16 & 26 & 3 & 45 & 0.4 \\
\hline \multicolumn{6}{|l|}{ Family history of DTC } \\
\hline Present & 7 & 8 & 1 & 16 & \\
\hline Absent & 13 & 21 & 1 & 35 & 0.7 \\
\hline \multicolumn{6}{|c|}{ Clinical presentation prior to surgery } \\
\hline Neck mass without compression & 17 & 27 & 2 & 46 & \\
\hline Neck mass with compression & 3 & 2 & 1 & 6 & 0.4 \\
\hline \multicolumn{6}{|l|}{ Local extension } \\
\hline Present & 9 & 12 & 1 & 22 & \\
\hline Absent & 11 & 16 & 2 & 29 & 0.9 \\
\hline \multicolumn{6}{|l|}{ Capsular invasion } \\
\hline Present & 11 & 12 & 3 & 26 & \\
\hline Absent & 9 & 16 & 0 & 25 & 0.1 \\
\hline \multicolumn{6}{|l|}{ Metastasis at presentation } \\
\hline Present & 12 & 8 & 0 & 20 & \\
\hline Absent & 8 & 21 & 3 & 32 & 0.02 \\
\hline
\end{tabular}

At the initial six-month follow-up, two patients (4\%) were deceased, and seven (13\%) were lost to follow up. Of 43 evaluable patients who underwent an initial assessment at six-month follow-up, $49 \%(n=21)$ were determined to have an incomplete ablation, and $51 \%(\mathrm{n}=22)$ were determined to have a complete ablation (Table 2). Of the 21 patients with incomplete ablation, $71 \%(\mathrm{n}=15)$ received a second dose of either $150 \mathrm{mCi}(5550 \mathrm{mBq})$ or $200 \mathrm{mCi}(7400 \mathrm{mBq})$ at 12 month follow-up, and $10 \%(\mathrm{n}=2)$ received a second dose at 18-month follow-up. Seven patients (32\%) received a third dose of $200 \mathrm{mci}(7400 \mathrm{mBq})$ at 18 months. At the end of two years, $39(75 \%)$ patients were evaluable for disease status and vital status (Table 3).

Table 2. Factors associated with outcomes at six months post RAI $(n=43)$.

\begin{tabular}{|c|c|c|c|c|c|}
\hline Category & Incomplete ablation & Complete ablation & Death & Total & p -value \\
\hline \multicolumn{6}{|l|}{ Metastasis at diagnosis } \\
\hline Present & 10 & 3 & 1 & 14 & \\
\hline Absent & 11 & 18 & 0 & 29 & $p=0.02$ \\
\hline \multicolumn{6}{|l|}{ Age } \\
\hline$>45$ & 9 & 11 & 0 & 20 & \\
\hline$<45$ & 12 & 10 & 1 & 23 & $p=0.52$ \\
\hline Male & 2 & 3 & 0 & 5 & \\
\hline Female & 19 & 18 & 1 & 38 & $p=0.83$ \\
\hline \multicolumn{6}{|l|}{ Clinical presentation } \\
\hline Neck mass without compression symptoms & 18 & 20 & 0 & 38 & \\
\hline Neck mass with compression symptoms & 3 & 1 & 1 & 5 & $p=0.01$ \\
\hline \multicolumn{6}{|l|}{ Type of surgery } \\
\hline Near total thyroidectomy & 18 & 13 & 0 & 31 & \\
\hline
\end{tabular}

\subsection{Factors Affecting Outcomes Post-Therapy}

Analysis of factors associated with incomplete ablation at six-month follow-up is summarized in Table 2. The presence of distant metastasis at diagnosis $(p=0.02)$ as well as the presence of compression symptoms associated with a neck mass $(p=0.01)$ were associated with incomplete ablation. An analysis of factors associated with incomplete ablation at two-year follow-up is summarized in Table 3 . The presence of metastasis(es) at diagnosis $(p=0.02)$, female gender $(p=0.02)$, and requirement for more than one RAI therapy $(p=0.00)$ were associated with incomplete ablation at 24 months follow-up. 
Table 3. Factors associated with outcomes at twenty four months post $R A I(N=39)$.

\begin{tabular}{|c|c|c|c|c|c|}
\hline Category & Incomplete ablation & Complete ablation & Death & Total & p-value \\
\hline \multicolumn{6}{|l|}{ Metastasis at diagnosis } \\
\hline Present & 6 & 5 & 1 & 12 & \\
\hline Absent & 5 & 22 & 0 & 27 & $p=0.02$ \\
\hline \multicolumn{6}{|l|}{ Age } \\
\hline$>45$ & 5 & 14 & 1 & 20 & \\
\hline$<45$ & 6 & 13 & 0 & 19 & $p=0.57$ \\
\hline \multicolumn{6}{|l|}{ Sex } \\
\hline Male & 2 & 2 & 1 & 5 & \\
\hline Female & 9 & 25 & 0 & 34 & $p=0.02$ \\
\hline \multicolumn{6}{|l|}{ Clinical presentation } \\
\hline Neck mass without compression & 8 & 26 & 1 & 25 & \\
\hline Neck mass with compression & 3 & 1 & 0 & 3 & $p=0.08$ \\
\hline \multicolumn{6}{|l|}{ Type of surgery } \\
\hline Near total thyroidectomy & 8 & 20 & 1 & 29 & \\
\hline Total thyroidectomy & 3 & 7 & 0 & 10 & $p=0.83$ \\
\hline \multicolumn{6}{|l|}{ Total RAI doses received } \\
\hline One & 3 & 20 & 1 & 24 & \\
\hline Two & 2 & 6 & 0 & 8 & \\
\hline Three & 6 & 1 & 0 & 7 & $P=0.00$ \\
\hline \multicolumn{6}{|c|}{ Cumulative amount of RAI Received in $\mathrm{mCi}$} \\
\hline 100 & 3 & 20 & 1 & 24 & \\
\hline 200 & 2 & 6 & 0 & 8 & \\
\hline 250 & 1 & 0 & 0 & 1 & \\
\hline$>300$ & 5 & 1 & 0 & 6 & $P=0.01$ \\
\hline
\end{tabular}

\section{Discussion}

This two years cohort study done at ORCI the only cancer center offering radioactive iodine treatment to DTC patients in Tanzania presents the outcome of patients treated who underwent post-surgical RAI therapy in Tanzania.

It shows that the occurrence of DTC between the age groups of below 45 and above 45 years of is equal. This is different from what is seen in other studies done elsewhere whereby most of DTC patients where $>45$ years(13). This could mean that the DTC in our setting occurs at a relatively younger age and maybe it is not age related. The longstanding endemic goiter which affects many areas of Tanzania [14] maybe the culprit.

Endemic goiter due to longstanding dietary iodine deficiency could also be the cause of the high occurrence of follicular and Hurthle cell histology subtypes [5]. Studies elsewhere have shown the relationship between dietary iodine deficiency and occurrence of follicular and Hurthle cell carcinomas [15]. Further we found out that the family history had low influence on occurrence unlike studies done elsewhere which could also be accounted by endemic goiter for decades [13]. Most of the patients who presented with metastasis were those with follicular subtypes. Follicular subtype is known for being more aggressive [16].

It is known that the more the extensive the surgery is the lower the chances of recurrence and hence the better the prognosis [17]. In this study most patients presented with goiter without compression symptoms. However about 50\% of patients had to go for second or third completion thyroidectomy before receiving RAI therapy since successful RAI treatment requires minimal or no thyroid remnant. This further delays RAI therapy and may contribute to poor outcome [18].

Outcome is influenced by pathological subtype whereby follicular subtype is more aggressive and associated with poor prognosis in comparison to papillary [18]. Similarly in this study, we found most patients with metastases at diagnosis were those with follicular subtype. Furthermore about $50 \%$ of patients had capsular invasion, a sign of advanced disease at diagnosis, which without aggressive post-surgical RAI therapy may lead to poor outcome.

All patients received the same ablation dose and assessment was done at 6 months to ascertain whether complete ablation has been achieved and factors contributing to it. It was evident that distant metastasis at diagnosis and clinical presentation were associated with poor outcome at six months. This is similar to what is seen elsewhere, whereby those patient who present with metastasis have up to $40 \% 10$ years survival while those who present without metastasis the 10 year survival is $>95 \%$ [19].

Looking at outcome at two years whereby those who had had incomplete ablation at six months received additional RAI therapy until complete ablation was achieved. The factors affecting outcome had increased whereby now we have metastasis at diagnosis, gender, number of RAI therapies and cumulative RAI therapies received. Male gender has been associated with more aggressive disease. We see gender being an important contributor of outcome at two years, and not immediately. But also repeated RAI therapies for those with incomplete ablation improves outcome, by attaining ablation after initial treatment [20].

This study gives an overall picture of thyroid DTC management in Tanzania. We saw 14 out of 52 patients had an advanced disease evidenced by distant metastasis on 
presentation. This contributes to poor clinical outcome by delaying to achieve complete ablation. We have seen about 15 out of 52 patients had to receive multiple therapies of RAI post repeated and completion surgery to achieve complete ablation. These multiple repeated doses translate to an extra financial burden to the patients, relatives and the government at large which could be significant in a limited resource setting.

This calls for increased awareness to the public and knowledge among clinicians of the importance of early detection, because in such patients complete ablation can be achieved by a single RAI ablation dose.

RAI is purchased and imported from South Africa (SA), where an ablation dose of $100 \mathrm{mCi}(3700 \mathrm{mBq})$ costs about 900USD which most patients cannot afford. Thanks to this study, the government is now aware of this cost implication to the vast majority of Tanzanian, consequently all public patients at ORCI now receive RAI paid for by the government. This further improves the treatment outcome of DTC patients since the patients are guaranteed of timely availability of RAI.

One of the limitations of this study was that patients are referred to ORCI after surgery has been done at either referral or regional hospitals because there are no surgical services at ORCI. Thus it is not possible to stage and ascertain the TNM status of the DTC prior to surgery and treatment; however assessment of metastasis was done at ORCI before RAI therapy. Another limitation was challenges of sending back patients with partial thyroidectomy for completion thyroidectomy because some had surgery in the regional or zonal hospitals far away from ORCI and refereeing them back was costly and the majority could not afford.

\section{Conclusion}

This study has shown that, the clinical outcome of DTC patients following RAI therapy at ORCI in Tanzania may be determined by presence of distant metastases at presentation, male sex, and extent of initial surgery.

\section{Recommendations}

The outcome of DTC patients can be improved by early diagnosis, and early referral for RAI post-surgery. This will allow complete ablation to be achieved by low RAI doses and short hospital stay resulting in reduced burden to the government and families for treating patients with DTC.

\section{Acknowledgements}

We acknowledge the cooperation we received from the ORCI administration and the nuclear medicine staff in particular during the whole study period.

\section{Funding}

The authors acknowledge the Tanzania Commission of Science and Technology (COSTECH) for financial support that made this study a success.

\section{Authors Contributions}

LS: designed the study, collected the data, made the analysis, wrote the manuscript

TM: supervised study designing, data collection, data analysis and manuscript writing

JM: assisted in study designing, data analysis and critical review

KM: supervised study designing, data collection, data analysis and manuscript writing

BS: supervised study designing, data collection, data analysis and manuscript writing

KVL: data analysis, manuscript writing, critical review

\section{References}

[1] Brown RL, de Souza JA, Cohen EE. Thyroid cancer: burden of illness and management of disease. J Cancer. 2011; 2: 193-9.

[2] Iarc. IA for R on CWHO. GLOBOCAN 2012: Estimated Cancer Incidence, Mortality and Prevalence Worldwide in 2012. Globocan. 2012.

[3] Schlumberger MJ. Papillary and Follicular Thyroid Carcinoma. N Engl J Med. 1998; 338 (5): 297-306.

[4] Htwe TT, Hamdi MM, Swethadri GK, Wong JOL, Soe MM, Abdullah MS. Incidence of thyroid malignancy among goitrous thyroid lesions from the Sarawak General Hospital 2000-2004. Singapore Med J. 2009; 50 (7): 724-8.

[5] Feldt-Rasmussen U. Iodine and Cancer. Thyroid. 2001; 11 (5): 483-6.

[6] Andrews W, Kaiser DL. ThyroidCancer: Some BasicConsiderations. 1983; 33 (2): 87-97.

[7] Handkiewicz-Junak D, Wloch J, Roskosz J, Krajewska J, Kropinska A, Pomorski L, et al. Total thyroidectomy and adjuvant radioiodine treatment independently decrease locoregional recurrence risk in childhood and adolescent differentiated thyroid cancer. J Nucl Med [Internet]. 2007; 48 (6): 879-88. Available from: http://www.ncbi.nlm.nih.gov/pubmed/17536106

[8] Manel J, Blesa G, Pulido EG, Pulla MP, Candel VA, Bautista $\mathrm{J}$, et al. Old and New Insights in the Treatment of Thyroid Carcinoma. 2010; 2010 (Table 1).

[9] Sawka AM, Thephamongkhol K, Brouwers M, Thabane L, Browman G, Gerstein HC. CLINICAL REVIEW 170 A Systematic Review and Metaanalysis of the Effectiveness of Radioactive Iodine Remnant Ablation for Well-Differentiated Thyroid Cancer. J Clin Endocrinol Metab. 2004; 89 (8): 3668-76.

[10] Silberstein EB, Alavi A, Balon HR, Clarke SEM, Divgi C, Gelfand MJ, et al. The SNMMI practice guideline for therapy of thyroid disease with 131I 3.0. J Nucl Med [Internet]. 2012 Oct [cited 2014 Oct 13]; 53 (10): 1633-51. Available from: http://www.ncbi.nlm.nih.gov/pubmed/22787108

[11] Cooper DS, Doherty GM, Haugen BR, Kloos RT, Lee SL, Mandel SJ, et al. Revised American Thyroid Association management guidelines for patients with thyroid nodules and differentiated thyroid cancer. Thyroid. 2009; 19: 1167214 . 
[12] Mazzaferri EL, Kloos RT. CLINICAL REVIEW 128 Current Approaches to Primary Therapy for Papillary and Follicular Thyroid Cancer. 2001; 86 (4): 1447-63.

[13] Passler C, Scheuba C. Prognostic factors of papillary and follicular thyroid cancer: differences in an iodine-replete endemic goiter region. Endocrine-Related ... [Internet]. 2004 [cited 2015 Jul 22]; 131-9. Available from: http://erc.endocrinology-journals.org/content/11/1/131.short

[14] Wachter W, Mvungi M, Konig A, Pickardt CR, Scriba PC. Prevalence of goitre and hypothyroidism in Southern Tanzania: Effect of iodised oil on thyroid hormone deficiency. J Epidemiol Community Health. 1986.

[15] Kalk WJ, Sitas F, Patterson AC. Thyroid cancer in South Africa--an indicator of regional iodine deficiency. S Afr Med J. 1997; 87 (6): 735-8.
[16] Sirveaux F, Brunaud L, Klein M, Muresan MM, Olivier P, Lecle J. Bone metastases from differentiated thyroid carcinoma. 2008; 37-49.

[17] Schlumberger MJ. Papillary and Follicular Thyroid Carcinoma. N Engl J Med. 1998; 338 (5): 297-306.

[18] Mazzaferri EL, Jhiang SM. Long-term impact of initial surgical and medical therapy on papillary and follicular thyroid cancer. Am J Med. 1994.

[19] Pacini F, Schlumberger M, Dralle H, Elisei R, Smit JWA, Wiersinga W, et al. European consensus for the management of patients with differentiated thyroid carcinoma of the follicular epithelium. Eur J Endocrinol. 2006.

[20] Haugen BR, Kane MA. Approach to the thyroid cancer patient with extracervical metastases. Journal of Clinical Endocrinology and Metabolism. 2010. 